\title{
Lactate level and unplanned readmission to the surgical intensive care unit: a retrospective cohort study
}

\author{
Tak Kyu Oh ${ }^{1 \#}$, Chami Im", In-Ae Song ${ }^{1}$ \\ ${ }^{1}$ Department of Anesthesiology and Pain Medicine, Seoul National University Bundang Hospital, Seongnam, Korea; ${ }^{2}$ Department of Surgery, Seoul \\ National University Bundang Hospital, Seongnam, Korea \\ Contributions: (I) Conception and design: TK Oh, C Im, IA Song; (II) Administrative support: IA Song, C Im; (III) Provision of study materials or \\ patients: TK Oh, C Im; (IV) Collection and assembly of data: TK Oh, IA Song; (V) Data analysis and interpretation: TK Oh, C Im; (VI) Manuscript \\ writing: All authors; (VII) Final approval of manuscript: All authors. \\ "These authors contributed equally to this work. \\ Correspondence to: In-Ae Song, MD, PhD. Department of Anesthesiology and Pain Medicine, Seoul National University Bundang Hospital, Gumi-ro \\ 173 Beon-gil, Bundang-gu, Seongnam 13620, Korea. Email: songoficu@outlook.kr.
}

\begin{abstract}
Background: This study aimed to investigate the association between lactate levels during intensive care unit (ICU) stay and unplanned ICU readmission rates within 48 and $120 \mathrm{~h}$ in surgical patients.

Methods: This single-center retrospective cohort study evaluated adult patients who were discharged from the ICU between January 2012 and December 2018. The association between lactate levels (initial lactate at ICU admission, peak level during ICU stay, and last level before ICU discharge) and unplanned ICU readmission was investigated using uni- and multivariable logistic regression analyses.

Results: The cohort included 3,707 patients. The unplanned ICU readmission rate within 48 and $120 \mathrm{~h}$ was $2.1 \%$ ( $\mathrm{n}=78$ patients) and $4.5 \%$ ( $\mathrm{n}=166$ patients), respectively. In the multivariable model, $1 \mathrm{mg} \mathrm{dL}$ increase in the peak lactate level during ICU stay was associated with 1.1-fold increase in unplanned ICU readmission within $48 \mathrm{~h}$ (odds ratio: 1.10; $95 \%$ confidence interval: 1.02 to $1.2 ; \mathrm{P}=0.016$ ). In contrast, both initial and last lactate levels during ICU stay were not significantly associated. A similar trend was observed for unplanned ICU readmission within $120 \mathrm{~h}$. On receiver operating characteristic analysis, the areas under the curve (AUC) of the initial, last, and peak lactate levels were $0.61,0.53$, and 0.63 , respectively.

Conclusions: The peak lactate level during ICU admission is independently associated with unplanned ICU readmission within both, 48 and $120 \mathrm{~h}$ among surgical ICU patients. However, the lactate level has relatively poor predictive capability for unplanned ICU readmissions, and a new model is needed to predict unplanned ICU readmissions among surgical patients.
\end{abstract}

Keywords: Critical care; hyperlactatemia; intensive care units (ICU); lactate; lactic acid

Submitted Jan 29, 2020. Accepted for publication Aug 10, 2020.

doi: 10.21037/apm-20-291

View this article at: http://dx.doi.org/10.21037/apm-20-291

\section{Introduction}

Intensive care unit (ICU) readmission after discharge is among the key markers of quality of care and outcomes in critically ill patients $(1,2)$. In previous studies, the ICU readmission rates ranged from $1.2 \%$ to $14.5 \%$ (3-6). ICU readmission is known to be associated with increased hospitalization and mortality rates among critically ill patients (3,4,7-10). Therefore, reducing ICU readmission rates has considerable implications with respect to the improvement of hospital outcomes, quality of care, hospital resource utilization, and cost savings in critically ill patients (11).

The serum lactate level, which was first reported by Broder and Weil et al. $(12,13)$, has been widely used as an important marker, that reflects altered tissue perfusion in 
critically ill patients (14). In particular, the elevation in lactate levels directly reflects tissue hypoperfusion induced by macrocirculatory or microcirculatory dysfunction $(15,16)$, reduced oxygen delivery $(17)$, enhanced glycolysis (18), or metabolic blocks of pyruvate dehydrogenase (19). Thus, elevated lactate levels or lactate kinetics is known to be a predictive factor for poor outcomes in critically ill patients (20). Previous studies have reported that elevated lactate levels were associated with higher mortality among surgical ICU patients (21) and neurologic ICU patients (22). Therefore, elevated lactate levels may serve as an indicator in predicting outcomes including ICU readmission among ICU patients. However, the outcome measure of these studies mostly included mortality and not ICU readmission, and thus, the association between lactate levels in the ICU and ICU readmission rates remains unclear.

Therefore, this study aimed to investigate the association between lactate levels (initial lactate at ICU admission, peak level during ICU stay, and last level before ICU discharge) and unplanned ICU readmission rates within 48 or $120 \mathrm{~h}$ in surgical ICU patients. We hypothesized that increased lactate levels during the ICU stay are associated with unplanned ICU readmission rates in surgical and critically ill patients.

We present the following article in accordance with the STROBE reporting checklist (available at http://dx.doi. org/10.21037/apm-20-291).

\section{Methods}

\section{Study design and data source}

The study was conducted in accordance with the Declaration of Helsinki (as revised in 2013). This retrospective cohort study was conducted in a single tertiary academic hospital and was approved by the Institutional Review Board (IRB) of Seoul National University Bundang Hospital (SNUBH) (IRB approval number: B-1904/534103). The requirement for informed consent was waived owing to the retrospective design of this study. Data were collected from the electronic medical records of Bundang Hospital Electronic System for Total Care (BESTCare) at SNUBH (23).

\section{Study population}

We evaluated adult patients ( $\geq 18$ years old) who were admitted to the ICU in the surgical department and were discharged from there between January 2012 and December 2018. For multiple ICU admissions $(\geq 2)$ in one patient, only the first ICU admission during the study period was included in the analysis, given that the main endpoint of this study was unplanned ICU readmission. Patients who were transferred to another hospital directly after ICU discharge and those with incomplete or missing medical records pertaining to serum lactate levels were excluded.

\section{Measurement of lactate level}

The lactate level was commonly measured using radial or femoral arterial blood in the ICU. The initial lactate level $\left(\mathrm{mg} \mathrm{dL}^{-1}\right)$ was defined as the first serum lactate level measured within 6 hours after ICU admission, regardless of whether the patient was admitted to the ICU from the emergency room, general ward, or postoperatively. The last lactate level was defined as the last measurement before ICU discharge. The maximum value of the lactate level during ICU stay was defined as the peak lactate level.

\section{Assessment of unplanned ICU readmission}

Unplanned ICU readmission was defined as an unexpected second admission to the ICU, from which the patient was originally discharged during the same hospitalization. We evaluated ICU readmission within 48 and $120 \mathrm{~h}$ based on previous studies $(5,24)$. We selected $48 \mathrm{~h}$, as proximate readmissions may be most likely to be attributable to ICU care or triage decisions (25). In contrast, readmissions after 120 hours are more likely to be elective or caused by events occurring on the floor after discharge.

\section{Potential covariates}

Clinicodemographic data, including sex, age (years), body mass index (BMI, $\mathrm{kg} \mathrm{m}^{-2}$ ), socioeconomic status [e.g., type of insurance (medical aid beneficiary program or national health insurance program)], Charlson comorbidity index at ICU admission, Acute Physiology and Chronic Health Evaluation (APACHE) II score at ICU admission, characteristics of ICU stay (year of discharge, duration, ventilator care, postoperative ICU care, and admission to the cardiothoracic department), were collected. Medical aid beneficiaries include those having low incomes; the bulk of the hospital charges for these patients are paid by the government. For patients in the national health insurance 
program, the government covers approximately two-thirds of their hospital charges. The International Classification of Disease-10 (ICD-10) codes in the electronic health record system were used to calculate the Charlson comorbidity index of all patients.

\section{Statistical analysis}

Baseline characteristics have been presented as the mean value with the standard deviation (SD) for continuous variables or numbers with percentages for categorical variables. Univariable logistic regression analysis was initially conducted to investigate the individual association of all covariates with ICU readmission. Covariates with a $\mathrm{P}$ value of $<0.2$ in the univariable model were then entered into the final multivariable logistic regression model. All the results of the logistic regression model were provided as odds ratio (OR) with $95 \%$ confidence interval (CI), and the goodness of fit in each multivariable model was confirmed via the Hosmer-Lemeshow statistic test with a criterion of $P>0.05$. We analyzed multi-collinearity in each multivariable model with a variance inflation factor $<2.0$. Finally, we performed receiver operating characteristic (ROC) analysis for unplanned readmission within $48 \mathrm{~h}$ based on three main variables (i.e., initial lactate, last lactate, and peak lactate level during ICU stay) with two traditional scoring systems, namely, APACHE II and Charlson comorbidity index. The results of the ROC analysis were presented as the areas under the curve (AUC) with 95\% CI. All analyses were performed using the $\mathrm{R}$ software package, version 3.6.0 (R Foundation for Statistical Computing, Vienna, Austria), and $\mathrm{P}<0.05$ was considered statistically significant.

\section{Results}

\section{Patient characteristics}

Among the 11,552 adult patients (age $\geq 18$ years) discharged alive from the surgical ICU between January 2012 and December 2018, 7,845 were excluded due to multiple admissions $(\mathrm{n}=712)$, transfer to another hospital after ICU discharge $(\mathrm{n}=114)$, and incomplete or missing medical records of serum lactate levels at ICU admission and discharge $(n=7,019)$. Finally, 3,707 patients were included in the analysis. The baseline patient characteristics are presented in Table 1. The mean (SD) initial, peak, and last lactate level $\left(\mathrm{mg} \mathrm{dL}^{-1}\right)$ after ICU admission were 2.7 (5.9), 4.2 (3.4), and 1.8 (1.2), respectively. The unplanned
ICU readmission rates within 48 and $120 \mathrm{~h}$ were $2.1 \%$ ( $\mathrm{n}=78$ patients) and $4.5 \%(\mathrm{n}=166$ patients), respectively (Figure 1). The departments with the highest readmission rates were the neurosurgery department $(53.6 \%)$, followed by cardiopulmonary $(23.8 \%)$, general surgery $(9.8 \%)$, and orthopedic surgery departments (5.6\%).

\section{Unplanned ICU readmissions and lactate level}

The mean peak lactate level was 6.66 and $4.13 \mathrm{mg} \mathrm{dL}^{-1}$ in those who were and were not readmitted within $48 \mathrm{~h}$, respectively, and was 5.55 and $4.11 \mathrm{mg} \mathrm{dL}^{-1}$ in those who were and were not readmitted within $120 \mathrm{~h}$, respectively. The results of uni- and multivariable logistic regression analyses for unplanned ICU readmission within $48 \mathrm{~h}$ after ICU discharge are presented in Table 2. In the multivariable logistic regression model for unplanned ICU readmission within $48 \mathrm{~h}$, a $1 \mathrm{mg} \mathrm{dL} \mathrm{d}^{-1}$ increase in peak lactate level during ICU stay was associated with a 1.1-fold increase in unplanned ICU readmission within 48 h (OR: 1.10; 95\% CI: 1.02 to $1.2 ; \mathrm{P}=0.016)$, but the initial and last lactate levels during ICU stay were not $(\mathrm{P}=0.336$ and $\mathrm{P}=0.342$, respectively). The results of uni- and multivariable logistic regression analyses for unplanned ICU readmission within $120 \mathrm{~h}$ after ICU discharge are presented in Table 3. In the multivariable logistic regression model for unplanned ICU readmission within $120 \mathrm{~h}$, a $1 \mathrm{mg} \mathrm{dL} \mathrm{dL}^{-1}$ increase in peak lactate level during ICU stay was associated with a 1.1-fold increase of unplanned ICU readmission within $120 \mathrm{~h}$ (OR: 1.10; $95 \%$ CI: 1.03 to $1.17 ; \mathrm{P}=0.002)$. However, both initial and last lactate levels during ICU stay were not significantly associated with unplanned ICU readmission within $120 \mathrm{~h}$ ( $\mathrm{P}=0.373$ and $\mathrm{P}=0.612$, respectively).

\section{Receiver operating characteristic analysis}

On ROC analysis for unplanned ICU readmissions within $48 \mathrm{~h}$, the AUCs of the initial, last, and peak lactate levels during ICU stay were 0.61 (95\% CI: 0.60 to 0.64$), 0.53$ (95\% CI: 0.51 to 0.55 ), and 0.63 (95\% CI: 0.60 to 0.65 ), respectively (Table 4). Additionally, the AUCs of the APACHE II score and Charlson comorbidity index were 0.62 (95\% CI: 0.60 to 0.64 ) and 0.55 (95\% CI: 0.53 to 0.57 ), respectively.

\section{Discussion}

The present study showed that in surgical ICU patients, 
Table 1 Baseline characteristics of patients

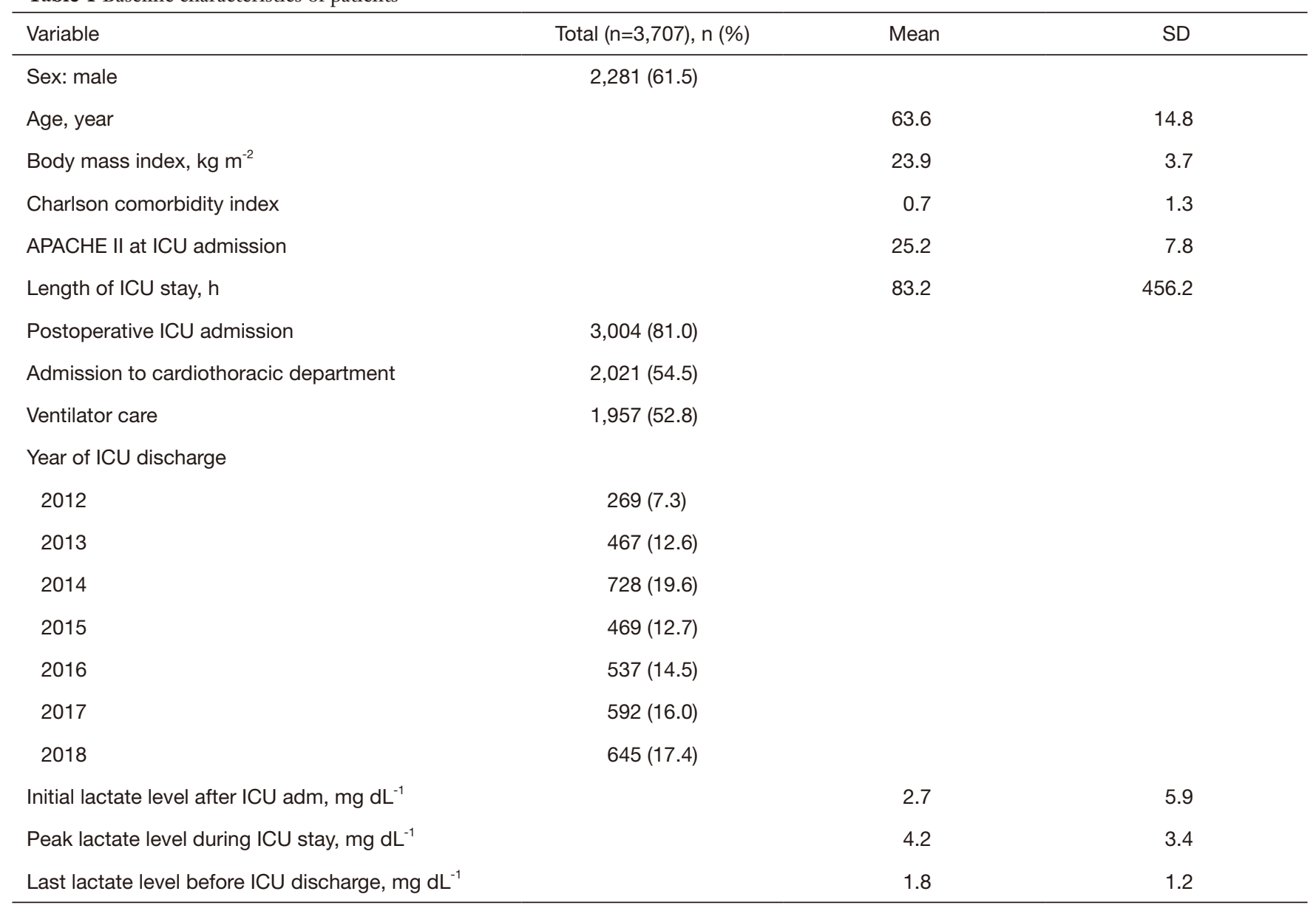

Presented as number (percentage) or mean value (standard deviation). SD, standard deviation; APACHE, acute physiology and chronic health evaluation; ICU, intensive care unit.

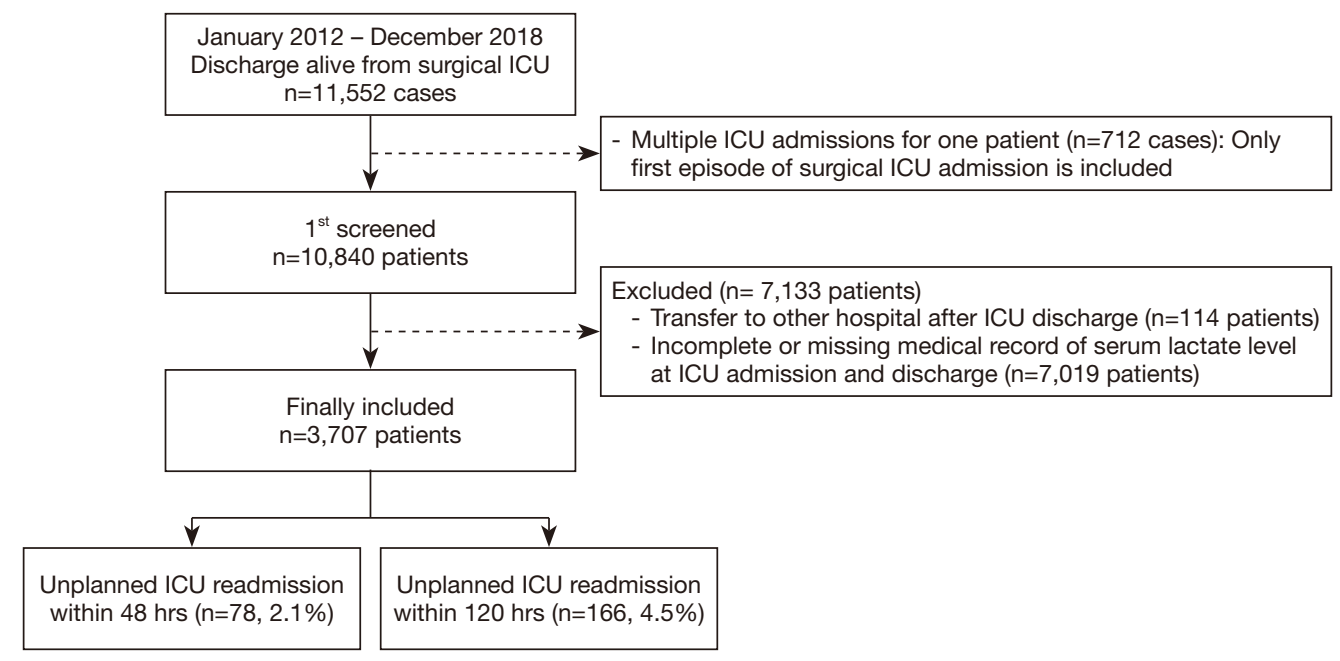

Figure 1 Patient selection flow chart. 
Table 2 Uni- and multivariable logistic regression analysis for unplanned ICU readmission within $48 \mathrm{~h}$ after ICU discharge

\begin{tabular}{|c|c|c|c|c|}
\hline Variable & \multicolumn{2}{|c|}{ Univariable model } & \multicolumn{2}{|c|}{ Multivariable model } \\
\hline Age, year & $1.02(1.00,1.04)$ & 0.014 & $1.02(1.00,1.04)$ & 0.040 \\
\hline Body mass index, $\mathrm{kg} \mathrm{m}^{-2}$ & $1.06(1.00,1.12)$ & 0.061 & $1.06(1.00,1.13)$ & 0.062 \\
\hline Male sex (vs. female) & $0.85(0.54,1.34)$ & 0.482 & & \\
\hline 2012 & 1 & & & \\
\hline 2013 & $1.06(0.39,2.89)$ & 0.913 & & \\
\hline 2014 & $0.86(0.33,2.26)$ & 0.759 & & \\
\hline 2015 & $0.96(0.34,2.66)$ & 0.930 & & \\
\hline 2018 & $1.26(0.49,3.21)$ & 0.630 & & \\
\hline Duration of ICU stay, per $10 \mathrm{~h}$ increase & $1.00(0.99,1.00)$ & 0.761 & & \\
\hline APACHE II at ICU admission, per 1 point increase & $1.04(1.01,1.07)$ & 0.008 & & \\
\hline Charlson comorbidity index, per 1 point increase & $1.12(0.96,1.30)$ & 0.141 & $1.12(0.95,1.31)$ & 0.181 \\
\hline Ventilator care during ICU stay (vs. no ventilator care) & $0.37(0.22,0.60)$ & $<0.001$ & $0.27(0.16,0.47)$ & $<0.001$ \\
\hline Postoperative ICU admission & $0.59(0.36,0.97)$ & 0.037 & $0.54(0.30,0.97)$ & 0.038 \\
\hline Admission to cardiothoracic department & $1.20(0.76,1.90)$ & 0.425 & & \\
\hline Initial lactate level after ICU adm, $1 \mathrm{mg} \mathrm{dL}^{-1}$ increase & $1.18(1.11,1.25)$ & $<0.001$ & $1.06(0.95,1.18)$ & 0.336 \\
\hline
\end{tabular}

Covariates with $\mathrm{P}<0.2$ were included in multivariable model for adjustment. Hosmer Lemeshow statistics of multivariable model: Chisquare, 10.67 ( $P=0.221)$. OR, odds ratio; $\mathrm{Cl}$, confidence interval; ICU, intensive care unit; APACHE, Acute Physiology and Chronic Health Evaluation.

the peak level of lactate during ICU stay was significantly associated with unplanned ICU readmission both, within 48 and $120 \mathrm{~h}$, but the initial lactate level after ICU admission or last lactate level before ICU discharge was not. These results indicate that the peak lactate level during ICU admission is the most appropriate predictor of unplanned ICU readmission among surgical patients. However, our results also showed that the lactate level during ICU stay has poor predictive capability (AUC $<0.7$ ), and cannot be used alone to predict unplanned ICU readmission.

The most important finding in this study was that among the initial, peak, and last lactate levels during ICU stay, only the peak lactate level was significantly associated with unplanned ICU readmission. This finding may reflect the characteristics of our surgical cohort. First, our cohort included $81.0 \%(3,004 / 3,707)$ of patients who were admitted to the surgical ICU postoperatively. A higher lactate level in the postoperative period during ICU stay could reflect postoperative complications $(26,27)$. Further, the peak lactate level in these patients may have reflected the severity of postoperative complications. In addition, a higher peak lactate level in these patients may have also reflected surgical stress (28), which may be affected by blood loss, surgical extent, or duration of surgery. Similarly, the peak lactate level was associated with postoperative outcomes such as mortality in cardiac surgery (29) or highrisk gastrointestinal surgery (30). Therefore, it is possible that in patients postoperatively admitted to the ICU, a 
Table 3 Uni- and multivariable logistic regression analysis for unplanned ICU readmission within $120 \mathrm{~h}$ after ICU discharge

\begin{tabular}{|c|c|c|c|c|}
\hline Variable & \multicolumn{2}{|c|}{ Univariable model } & \multicolumn{2}{|c|}{ Multivariable model } \\
\hline Age, year & $1.02(1.01,1.03)$ & 0.001 & $1.02(1.00,1.03)$ & 0.011 \\
\hline Body mass index, $\mathrm{kg} \mathrm{m}^{-2}$ & $0.99(0.95,1.04)$ & 0.775 & & \\
\hline Male sex (vs. female) & $1.05(0.76,1.45)$ & 0.762 & & \\
\hline 2012 & 1 & & 1 & \\
\hline 2013 & $1.78(0.86,3.70)$ & 0.123 & $2.64(1.17,5.94)$ & 0.019 \\
\hline 2014 & $1.19(0.58,2.46)$ & 0.637 & $1.98(0.88,4.43)$ & 0.097 \\
\hline 2015 & $0.97(0.44,2.16)$ & 0.949 & $1.44(0.60,3.45)$ & 0.413 \\
\hline 2018 & $1.58(0.77,3.22)$ & 0.211 & $2.59(1.15,5.81)$ & 0.021 \\
\hline Duration of ICU stay, per $10 \mathrm{~h}$ increase & $1.01(1.00,1.01)$ & 0.441 & & \\
\hline APACHE II at ICU admission, per 1 point increase & $1.02(1.00,1.04)$ & 0.151 & $1.02(1.00,1.04)$ & 0.099 \\
\hline Charlson comorbidity index, per 1 point increase & $1.08(0.97,1.21)$ & 0.159 & $1.04(0.92,1.17)$ & 0.535 \\
\hline Ventilator care during ICU stay (vs. no ventilator care) & $0.42(0.30,0.58)$ & $<0.001$ & $0.34(0.24,0.48)$ & $<0.001$ \\
\hline Postoperative ICU admission & $0.53(0.37,0.74)$ & $<0.001$ & $0.48(0.33,0.70)$ & $<0.001$ \\
\hline Admission to cardiothoracic department & $1.09(0.80,1.50)$ & 0.577 & & \\
\hline Initial lactate level after ICU adm, $1 \mathrm{mg} \mathrm{dL}^{-1}$ increase & $1.13(1.08,1.19)$ & $<0.001$ & $1.04(0.97,1.13)$ & 0.373 \\
\hline
\end{tabular}

Covariates with $\mathrm{P}<0.2$ were included in multivariable model for adjustment. Hosmer Lemeshow statistics: Chi-square, 10.47 ( $\mathrm{P}=0.233)$. OR, odds ratio; $\mathrm{Cl}$, confidence interval; ICU, intensive care unit; APACHE, Acute Physiology and Chronic Health Evaluation.

higher peak lactate level during ICU admission could reflect both, postoperative complications and surgical stress, and this could influence unplanned ICU readmission.

However, our results showed that ICU admission in the postoperative period or mechanical ventilator use was associated with a lower rate of unplanned readmission compared with other patients in the surgical department or with no use of mechanical ventilators. This finding may have been influenced by the characteristics of the study population. Our cohort only involved patients who were discharged from the ICU to the ward and excluded those who were severely ill or needed palliative care, and not ICU management (i.e., those who died in ICU, those who were transferred to another hospital, or those who agreed to sign a do-not-resuscitate form). Therefore, the results on postoperative ICU admission or mechanical ventilator use in the ICU need to be interpreted carefully, because these factors were not causal factors for lowering unplanned readmission rates, but reflected the characteristics of the study population

In our cohort, $19.0 \%(703 / 3,707)$ of the patients were not postoperatively admitted to the ICU but were rather admitted from the emergency department or ward. While many patients who undergo elective surgery are postoperatively admitted to the ICU only for continuous monitoring (31), surgical patients who are admitted to the ICU from the emergency department or ward may have more severe illnesses such as sepsis or bleeding. In these patients, the peak lactate level may reflect the disease severity, since an elevated lactate level is a useful marker 
Table 4 Receiver operating characteristics analysis for unplanned readmission within $48 \mathrm{~h}$ according to lactate level $\left(\mathrm{mg} \mathrm{dL}^{-1}\right)$

\begin{tabular}{ll}
\hline Variable & AUC $(95 \% \mathrm{Cl})$ \\
\hline Initial lactate level after ICU admission & $0.61(0.60,0.64)$ \\
Last lactate level before ICU discharge & $0.53(0.51,0.55)$ \\
Peak lactate level during ICU stay & $0.63(0.60,0.65)$ \\
APACHE II & $0.62(0.60,0.64)$ \\
Charlson comorbidity index & $0.55(0.53,0.57)$
\end{tabular}

AUC, area under the curve; ROC, receiver operating characteristics; ICU, intensive care unit; $\mathrm{Cl}$, confidence interval; APACHE, Acute Physiology and Chronic Health Evaluation.

for the severity of acute illness in critically ill patients (32). Therefore, the peak lactate level during ICU stay in this cohort reflects not only the severity of critical illness, but also predicts unplanned ICU readmission.

A recent study by Martin $e t$ al. suggested that a validated clinical nomogram, which was derived from seven physiologic and demographic variables, could predict unplanned ICU readmission within $72 \mathrm{~h}$ among surgical patients (33). In their study, the clinical nomogram had an AUC of 0.71 for predicting unplanned ICU readmission within $72 \mathrm{~h}$, which is relatively higher than that of the peak lactate level (0.63) or initial lactate level (0.61) in our study. However, the clinical nomogram was validated using seven physiologic and demographic variables (33), and we only used one variable, that is, lactate levels. Thus, if the lactate level during ICU admission is combined with other variables for developing a new model for predicting the risk of unplanned ICU admission, this model will have more predictive power. Future studies are needed to validate a new model or scoring system for predicting unplanned ICU readmission among surgical patients.

This study has some limitations. First, because this was retrospective cohort study, there were several confounders that should be controlled. Second, the generalizability of our findings may be limited, because the study was conducted in a single center. Third, many patients $(7,019$ patients) were excluded because their lactate level was not measured, and this could have affected the patient characteristics. For instance, relatively healthy patients whose lactate level did not need to be assessed during ICU admission may have been excluded from the final analysis. Lastly, the timing of measurement of lactate levels differed, and this could have led to bias.
In conclusion, this study showed that in surgical ICU patients, the peak lactate level during ICU admission was independently associated with unplanned ICU readmission within both, 48 and $120 \mathrm{~h}$. However, the lactate level alone has relatively poor predictive capability for unplanned ICU readmission, and thus, a new model is needed.

\section{Acknowledgments}

Funding: None.

\section{Footnote}

Reporting Checklist: The authors have completed the STROBE reporting checklist. Available at http://dx.doi. org/10.21037/apm-20-291

Data Sharing Statement: Available at http://dx.doi. org/10.21037/apm-20-291

Conflicts of Interest: All authors have completed the ICMJE uniform disclosure form (available at http://dx.doi. org/10.21037/apm-20-291). The authors have no conflicts of interest to declare.

Ethical Statement: The authors are accountable for all aspects of the work in ensuring that questions related to the accuracy or integrity of any part of the work are appropriately investigated and resolved. The study was conducted in accordance with the Declaration of Helsinki (as revised in 2013). This retrospective cohort study was conducted in a single tertiary academic hospital and was approved by the Institutional Review Board (IRB) of Seoul National University Bundang Hospital (SNUBH) (IRB approval number: B-1904/534-103). The requirement for informed consent was waived owing to the retrospective design of this study.

Open Access Statement: This is an Open Access article distributed in accordance with the Creative Commons Attribution-NonCommercial-NoDerivs 4.0 International License (CC BY-NC-ND 4.0), which permits the noncommercial replication and distribution of the article with the strict proviso that no changes or edits are made and the original work is properly cited (including links to both the formal publication through the relevant DOI and the license). See: https://creativecommons.org/licenses/by-nc-nd/4.0/. 


\section{References}

1. Berenholtz SM, Dorman T, Ngo K, et al. Qualitative review of intensive care unit quality indicators. J Crit Care 2002;17:1-12.

2. Rhodes A, Moreno RP, Azoulay E, et al. Prospectively defined indicators to improve the safety and quality of care for critically ill patients: a report from the Task Force on Safety and Quality of the European Society of Intensive Care Medicine (ESICM). Intensive Care Med 2012;38:598-605.

3. Elliott M, Worrall-Carter L, Page K. Intensive care readmission: a contemporary review of the literature. Intensive Crit Care Nurs 2014;30:121-37.

4. Kramer AA, Higgins TL, Zimmerman JE. The association between ICU readmission rate and patient outcomes. Crit Care Med 2013;41:24-33.

5. Brown SE, Ratcliffe SJ, Kahn JM, et al. The epidemiology of intensive care unit readmissions in the United States. Am J Respir Crit Care Med 2012;185:955-64.

6. Al-Jaghbeer MJ, Tekwani SS, Gunn SR, et al. Incidence and Etiology of Potentially Preventable ICU Readmissions. Crit Care Med 2016;44:1704-9.

7. de Lima VC, Bierrenbach AL, Alencar GP, et al. Increased risk of death and readmission after hospital discharge of critically ill patients in a developing country: a retrospective multicenter cohort study. Intensive Care Med 2018;44:1090-6.

8. Jeong BH, Na SJ, Lee DS, et al. Readmission and hospital mortality after ICU discharge of critically ill cancer patients. PLoS One 2019;14:e0211240.

9. Kareliusson F, De Geer L, Tibblin AO. Risk prediction of ICU readmission in a mixed surgical and medical population. J Intensive Care 2015;3:30.

10. Ponzoni CR, Correa TD, Filho RR, et al. Readmission to the Intensive Care Unit: Incidence, Risk Factors, Resource Use, and Outcomes. A Retrospective Cohort Study. Ann Am Thorac Soc 2017;14:1312-9.

11. Kramer AA, Higgins TL, Zimmerman JE. Intensive care unit readmissions in U.S. hospitals: patient characteristics, risk factors, and outcomes. Crit Care Med 2012;40:3-10.

12. Broder G, Weil MH. Excess Lactate: An Index of Reversibility of Shock in Human Patients. Science 1964;143:1457-9.

13. Weil MH, Afifi AA. Experimental and clinical studies on lactate and pyruvate as indicators of the severity of acute circulatory failure (shock). Circulation 1970;41:989-1001.

14. Vincent JL, De Backer D. Circulatory shock. N Engl J
Med 2013;369:1726-34.

15. James JH, Luchette FA, McCarter FD, et al. Lactate is an unreliable indicator of tissue hypoxia in injury or sepsis. Lancet 1999;354:505-8.

16. Jones AE, Puskarich MA. Sepsis-induced tissue hypoperfusion. Crit Care Clin 2009;25:769-79, ix.

17. Kompanje EJ, Jansen TC, van der Hoven B, et al. The first demonstration of lactic acid in human blood in shock by Johann Joseph Scherer (1814-1869) in January 1843. Intensive Care Med 2007;33:1967-71.

18. Rogatzki MJ, Ferguson BS, Goodwin ML, et al. Lactate is always the end product of glycolysis. Front Neurosci 2015;9:22.

19. Heigenhauser GJ, Parolin ML. Role of pyruvate dehydrogenase in lactate production in exercising human skeletal muscle. Adv Exp Med Biol 1999;474:205-18.

20. Vincent JL, Quintairos ESA, Couto L Jr, et al. The value of blood lactate kinetics in critically ill patients: a systematic review. Crit Care 2016;20:257.

21. Oh TK, Song IA, Jeon YT. Peri-operative serum lactate level and postoperative 90-day mortality in a surgical ICU: A retrospective association study. Eur J Anaesthesiol 2020;37:31-7.

22. Oh TK, Song IA, Bae HJ, et al. Serum lactate level upon admission to the neuro-intensive care unit and 90day mortality: A retrospective study. J Clin Neurosci 2019;70:173-7.

23. Yoo S, Lee KH, Lee HJ, et al. Seoul National University Bundang Hospital's electronic system for total care. Healthc Inform Res 2012;18:145-52.

24. Woodhouse D, Berg M, van der Putten J, et al. Will benchmarking ICUs improve outcome? Curr Opin Crit Care 2009;15:450-5.

25. SCCM Quality Indicators Committee. Candidate Critical Care Quality Indicators. Anaheim. CA, Society of Critical Care Medicine 1995.

26. Hajjar LA, Almeida JP, Fukushima JT, et al. High lactate levels are predictors of major complications after cardiac surgery. J Thorac Cardiovasc Surg 2013;146:455-60.

27. Li S, Peng K, Liu F, et al. Changes in blood lactate levels after major elective abdominal surgery and the association with outcomes: a prospective observational study. J Surg Res 2013;184:1059-69.

28. Marnitz U, Dauberschmidt R, Mrochen H. The value of blood lactate determination in the postoperative phase. Anaesthesiol Reanim 1994;19:103-9.

29. Haanschoten MC, Kreeftenberg HG, Arthur Bouwman $\mathrm{R}$, et al. Use of Postoperative Peak Arterial Lactate Level 
to Predict Outcome After Cardiac Surgery. J Cardiothorac Vasc Anesth 2017;31:45-53.

30. Creagh-Brown BC, De Silva AP, Ferrando-Vivas P, et al. Relationship Between Peak Lactate and Patient Outcome Following High-Risk Gastrointestinal Surgery: Influence of the Nature of Their Surgery: Elective Versus Emergency. Crit Care Med 2016;44:918-25.

31. Kahan BC, Koulenti D, Arvaniti K, et al. Critical care admission following elective surgery was not associated with survival benefit: prospective analysis of data from 27

Cite this article as: Oh TK, Im C, Song IA. Lactate level and unplanned readmission to the surgical intensive care unit: a retrospective cohort study. Ann Palliat Med 2021;10(2):1012-1020. doi: 10.21037/apm-20-291 countries. Intensive Care Med 2017;43:971-9.

32. Kushimoto S, Akaishi S, Sato T, et al. Lactate, a useful marker for disease mortality and severity but an unreliable marker of tissue hypoxia/hypoperfusion in critically ill patients. Acute Med Surg 2016;3:293-97.

33. Martin LA, Kilpatrick JA, Al-Dulaimi R, et al. Predicting ICU readmission among surgical ICU patients: Development and validation of a clinical nomogram. Surgery 2019;165:373-80. 